\title{
BreastCare
}

\section{Palbociclib - from Bench to Bedside and Beyond}

\author{
Marcus Schmidt \\ Department of Obstetrics and Gynecology, Johannes Gutenberg University, Mainz, Germany
}

\author{
Keywords \\ Breast cancer - Estrogen receptor - CDK4/6 - Palbociclib . \\ PD 0332991
}

\section{Summary}

Endocrine therapy is the cornerstone in the treatment of hormone receptor-positive breast cancer. During the last decades, much has been learned about the subtle regulation of the cell cycle. In this tightly regulated network, cyclin-dependent kinases (CDKs) play a pivotal role. Especially CDK4/6 is the key regulator of the G1-S transition. Realizing its importance, specific inhibitors of CDK4/6 were developed. The drug most advanced in clinical development in this class is palbociclib (PD 0332991). This review highlights preclinical data and brings into focus early clinical trials that led to an accelerated approval by the US Food and Drug Administration (FDA) as first-line treatment in combination with letrozole in advanced hormone receptor-positive and human epidermal growth factor receptor 2 (HER2)-negative breast cancer. Furthermore, ongoing clinical trials with palbociclib in advanced and in early breast cancer are outlined. In conclusion, inhibition of CDK4/6 using palbociclib in combination with endocrine therapy is an efficacious treatment option in hormone receptor-positive/HER2-negative advanced breast cancer. Ongoing clinical trials will show whether palbociclib is ready for prime time in early breast cancer.

(C) 2016 S. Karger GmbH, Freiburg

\section{Bench - Cell Cycle Regulation and Preclinical Research with PD 0332991 (Palbociclib)}

Evasion of growth suppressors with disrupted cell cycle regulation is one of the hallmarks of cancer [1]. Indeed, tumor cells are evading growth suppressors and circumvent programs that negatively regulate cell proliferation. This negative regulation is largely maintained by tumor suppressor genes like $R B$ (retinoblastoma). The retinoblastoma protein $(\mathrm{Rb})$ is of critical importance for the transmission of growth-inhibitory signals originating outside the cell. Hence, $\mathrm{Rb}$ is a critical gatekeeper of cell cycle progression whose absence permits persistent cell proliferation. This wellknown hallmark of cancer can be targeted with drugs interfering with the regulation of the cell cycle.

\section{Regulation of the Cell Cycle}

Cells must progress through the four phases of the cell cycle to divide and replicate: G1, S phase (DNA synthesis), G2, and M phase (mitosis). The central players for regulation of the cell cycle are the cyclin-dependent kinases (CDKs), a group of serine/threonine kinases that form active heterodimeric complexes following binding to cyclins, thereby facilitating the transitions of the cell cycle [2]. CDK4/6 is the key regulator of the G1-S transition. In complex with cyclin $\mathrm{D}, \mathrm{CDK} 4 / 6$ phosphorylates $\mathrm{Rb}$ and drives cell cycle progression, a process inhibited by p16 (fig. 1). In its active hypophosphorylated state, Rb inhibits the transcription factor E2F, which is of pivotal importance for the progression from G1 to the $S$ phase (reviewed in [3]). In response to mitogenic signals, cells synthesize cyclin D, which then forms a complex with CDK4/6, leading to the phosphorylation of $\mathrm{Rb}$. When $\mathrm{Rb}$ is phosphorylated, the inhibition of the transcription factor E2F, which leads to progression of cells through the cell cycle, is lost. However, cell cycle regulation has a lot of built-in redundancy. This means that cells can proliferate even in the absence of CDK4/6 by using CDK2 instead. Transcription of the cyclin $\mathrm{E}$ gene serves as a positive feedback loop, because cyclin $\mathrm{E}$ forms a complex with CDK2. Either way, it 


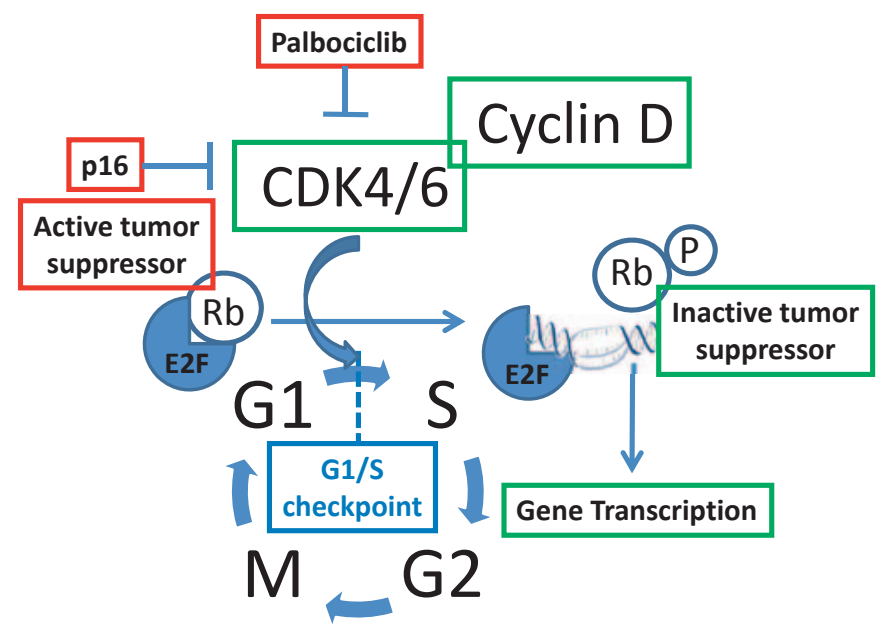

Fig. 1. Role of $C D K 4 / 6$ in cell cycle regulation. $C D K=$ Cyclin-dependent kinase, $\mathrm{E} 2 \mathrm{~F}=\mathrm{E} 2$ promoter binding factor, $\mathrm{G} 1=$ gap 1 phase, $\mathrm{G} 2=$ gap 2 phase, $\mathrm{M}=$ mitotic phase, $\mathrm{P}=$ phosphorylation, $\mathrm{p} 16$ = protein $16 /$ cyclin-dependent kinase inhibitor $2 \mathrm{~A}$ /multiple tumor suppressor $1, \mathrm{Rb}=$ retinoblastoma protein, $\mathrm{S}=$ synthetic phase.

leads to phosphorylation of $\mathrm{Rb}$, thus disrupting the binding of $\mathrm{Rb}$ to E2F, which allows E2F activation and the transcription of genes necessary for $\mathrm{S}$ phase entry and cell cycle progression. However, there are also negative regulators of cell cycle progression. As cells age, p16 plays a major role in the induction of cellular senescence in breast cells [4]. p16 is of critical importance, because it inhibits CDK4/6 and leads to the release and degradation of cyclin D as well as to a redistribution of p21 and p27 to cyclin E/CDK2, thus contributing to G1 arrest. Additionally, anti-proliferative signals induce p21 and p27, thereby also inhibiting the cyclin E/CDK2 complex and leading to G1 arrest. However, this tightly regulated pathway is frequently disrupted in breast cancer through p16 loss, CDK4/6 amplification, cyclin D overexpression, or Rb loss, leading to inactivation of the G1-to-S phase checkpoint [3].

\section{Preclinical Research with PD 0332991 (Palbociclib)}

The well-known frequent activation of the pathway described above in cancer led to efforts to block it pharmacologically [5]. The first-generation CDK inhibitors like flavopiridol were nonselective CDK inhibitors that blocked CDK4 but also had significant side effects. However, in recent years, several selective CDK4/6 inhibitors were developed and have entered clinical studies. The most advanced drug in this class is PD 0332991 or palbociclib (Pfizer, Inc.).

Palbociclib is a potent oral inhibitor of CDK4 and CDK6 that prevents the downstream phosphorylation of $\mathrm{Rb}$, thereby leading to G1 arrest. Fry et al. [6] showed that oral administration of PD 0332991 to mice produced marked tumor regression in a broad spectrum of human tumor xenografts in vivo. Additionally, they showed that PD 0332991 led to the elimination of phospho-Rb and the proliferation marker Ki-67 in tumor tissue as well as to the down-regulation of genes under the transcriptional control of E2F. Given the high efficacy and specificity of PD 0332991, this drug was scheduled early for clinical trials to answer the question whether selective inhibitors of CDK4/6 can provide a therapeutic benefit in cancer patients.

In further preclinical investigations, it was shown that $\mathrm{PD}$ 0332991 achieved specific inhibition of CDK4/6 and induced G1 arrest in primary bone marrow myeloma cells ex vivo, and prevented tumor growth in disseminated human myeloma xenografts [5]. Furthermore, the authors showed that, when used in combination with a second agent, the efficacy of PD 0332991 was markedly increased.

Building on these early results showing the efficacy of PD 0332991 in myeloma cells, Menu et al. [7] reported that the combination of PD 0332991 with bortezomib, a proteasome inhibitor widely used in myeloma treatment, increased tumor suppression and survival in the immunocompetent 5T33MM myeloma model. Indeed, induction of G1 arrest by PD 0332991 sensitized 5T33MM tumor cells to killing by bortezomib. The authors speculated that this combination therapy should prove useful in myeloma patients.

However, alterations of CDK4/6 are important not only for malignant myeloma but also for a variety of other types of cancer, including breast cancer. Finn et al. [8] described in their seminal preclinical work the in vitro sensitivity to PD 0332991 across a panel of molecularly characterized human breast cancer cell lines. They used 47 breast cancer cell lines representing known molecular subtypes (i.e. luminal, HER2 overexpressing, basal) and determined the $\mathrm{IC}_{50}$ value (concentration resulting in half-maximal inhibition) for each cell line. These results were then compared with gene expression data of these cell lines to characterize genes associated with in vitro sensitivity to PD 0332991. In general, estrogen receptor (ER)-positive luminal cell lines showed the highest sensitivity. This was also true when HER2 was amplified. In contrast, ER-negative basal cell lines displayed the least sensitivity. Using gene expression analysis (Agilent Human 1A V1), Finn et al. [8] identified 450 differentially expressed genes between sensitive and non-sensitive cell lines. 253 genes were upregulated in sensitive cell lines and 197 genes were upregulated in resistant lines. Sensitivity correlated positively with increased Rb and cyclin D1 (CCND1) expression as well as decreased p16 expression. This pattern of expression is in accordance with the mechanism of CDK4/6 inhibition as outlined above. As expected, cell cycle analyses revealed a G0/1 arrest in sensitive cell lines, and Western blot analyses demonstrated that phosphorylation of $\mathrm{Rb}$ was blocked in sensitive cell lines. In addition, Finn et al. [8] could demonstrate that the combination of PD 0332991 with tamoxifen in ER-positive cell lines and with trastuzumab in HER2-amplified cell lines showed a strong synergism in vitro. Moreover, treatment of a cell line with acquired resistance to tamoxifen with PD 0332991 reversed this resistance, either when PD 0332991 was used as a monotherapy or when these 2 agents were used in combination. The authors concluded their comprehensive preclinical investigations with the notion of a strong rationale for clinical development of PD 0332991 focusing on ER-positive luminal and on HER2-amplified breast cancer. Furthermore, they proposed a combination of PD 0332991 with anti-estrogen or antiHER2 therapy, respectively [8]. Conversely, in another preclinical 
study, PD 0332991 decreased the efficacy of DNA-damaging cytotoxic drugs like carboplatin in breast cancer xenografts in mice [9]. In line with these findings, well-known toxicities of carboplatin like thrombocytopenia were ameliorated when mice were also treated with PD 0332991. Thus, the authors concluded that CDK4/6 inhibitors should not be combined with DNA-damaging therapies in tumors that require CDK4/6 activity for proliferation.

\section{Bedside - Development of Palbociclib (PD 0332991) from Phase 1 to Accelerated Approval by the US Food and Drug Administration}

To proceed with the clinical development of this drug, 2 phase 1 trials were undertaken. In the first-in-human phase 1 study, Schwartz et al. [10] enrolled 33 patients with Rb-positive advanced solid tumors or non-Hodgkin's lymphoma refractory to standard therapy. Patients received PD 0332991 once daily for 14 days followed by 7 days off treatment. The maximum tolerated dose (MTD) was $200 \mathrm{mg}$ once daily. The drug was slowly absorbed and eliminated with a mean half-life of $26.7 \mathrm{~h}$, resulting in its accumulation following repeated dosing. Extensive penetration of peripheral tissue was suggested by the large volume of distribution (mean 3.241 l). Adverse events were generally mild to moderate. Doselimiting toxicities (DLT) were mostly myelosuppression. In this phase 1 trial, early signs of efficacy were noticed with 1 partial remission and 9 patients with stable disease (SD). Taken together, the authors recommended this $200 \mathrm{mg} / \mathrm{d} 2$ weeks-on and 1 week-off $(2 / 1)$ schedule for further development.

A second phase I trial investigated the DLT and MTD of the first-in-class oral CDK4/6 inhibitor PD 0332991 administered once daily for 21 of 28 days ( $3 / 1$ schedule) in patients with $\mathrm{Rb}$-positive advanced solid tumors, and described pharmacokinetic-pharmacodynamic relationships relative to drug effects [11]. Flaherty et al. [11] enrolled 41 patients, including 5 with breast cancer, in this open-label phase I trial with standard $3+3$ design, with the provision for cohort expansion to 6 evaluable patients if a DLT was observed in the first cycle of treatment among the initial 3 patients. Similar to the phase I trial reported by Schwartz et al. [10], PD 0332991 was slowly absorbed (median T(max), $5.5 \mathrm{~h}$ ), and slowly eliminated (mean half-life of $25.9 \mathrm{~h}$ ) with a large volume of distribution (mean, 2.793 l). The MTD and recommended phase 2 dose of PD 0332991 was $125 \mathrm{mg}$ once daily. Neutropenia was the only dose-limiting effect with $12 \%$ grade 3 neutropenia after cycle 1 . The pharmacodynamics for absolute neutrophile count (ANC) and platelet levels showed a nadir occurring at the end of the dosing period in cycles 1 and 2 for both cell types, with a rebound of both the ANC and platelet levels during the off-drug period that continued up to day 8 of the following dosing cycle. Other adverse events were mostly mild to moderate and included fatigue (34\%), nausea (24\%), and diarrhea (15\%) with only few grade 3 events. The drug showed signs of clinical efficacy in this heavily pretreated group of patients with SD after $\geq 4$ cycles in $27 \%$. Taken together, this phase 1 study fulfilled its primary objective of establishing the safety pro- file of PD 0332991 and identifying a recommended 3/1 schedule dose for further investigation in phase 2 studies.

Considering the proposed mechanism of action with $\mathrm{Rb}$ as a potential biomarker, 37 patients with advanced breast cancer with positive $\mathrm{Rb}$ protein were enrolled in a single-arm phase 2 trial [12]. Patients were treated with single-agent palbociclib $125 \mathrm{mg}$ daily in a 3/1 schedule as proposed in a preceding phase 1 trial [11]. Primary objectives were tumor response and tolerability. Secondary objectives included progression-free survival (PFS) and a comprehensive biomarker assessment including $\mathrm{Rb}$ expression/localization, KI-67, p16 loss, and cyclin D amplification. The majority of the patients (84\%) were hormone receptor positive and HER2 negative, $5 \%$ were hormone receptor positive with coexpression of HER2, and $11 \%$ were triple negative. Patients had received a median of 2 prior cytotoxic therapies. Palbociclib showed clinical activity in this heavily pretreated cohort of breast cancer patients, with an overall clinical benefit rate of $19 \%$. The median PFS overall was 3.7 months, but significantly longer for those with hormone receptor-positive versus -negative disease $(\mathrm{P}=0.03)$ and for those who had previously progressed through endocrine therapy for advanced disease $(P=0.02)$. The major toxicity was neutropenia ( $51 \%$ grade $3 / 4$ ), which could be easily managed with dose reduction. Contrary to the assumed mechanism of action as outlined above, the analysis results of neither p16 nor cyclin D were significantly associated with clinical benefit or PFS. The authors concluded that palbociclib demonstrated single-agent activity in this heavily pretreated population of patients with advanced breast cancer.

Building on the encouraging results of palbociclib in hormone receptor-positive breast cancer, an open-label randomized phase 2 trial enrolled 165 postmenopausal ER-positive and HER2-negative breast cancer patients who had not received any systemic therapy for advanced disease (PALOMA-1) [13]. Patients were randomized either to letrozole alone or to letrozole + palbociclib (125 mg, given once daily for 3 weeks, followed by 1 week off over 28-day cycles). Patients were enrolled sequentially in 2 separate cohorts: In cohort 1, patients were enrolled on the basis of their ER-positive and HER2-negative biomarker status alone, whereas in cohort 2 they were also required to have amplification of CCND1 or loss of p16. Surprisingly, preliminary results from cohort 1 suggested that further patient selection based on CCND1 amplification or p16 loss was unlikely to further improve the patient outcome over the use of ER and HER2 status alone (hazard ratio (HR) with CCND1 or p16 copy changes $0.37 ; 95 \%$ confidence interval (CI) $0.10-1.40 ; \mathrm{p}=$ 0.13 vs. HR with no CCND1 or p16 copy changes $0.19 ; 95 \%$ CI $0.05-0.67 ; p=0.0045)$. For the whole cohort of patients, the median PFS was 10.2 months for the letrozole group and 20.2 months for the palbociclib + letrozole group (HR 0.488, 95\% CI 0.319$0.748 ; \mathrm{p}=0.0004)$. Grade $3 / 4$ neutropenia was reported in $54 \%$ in the palbociclib + letrozole group versus $1 \%$ in the letrozole group. However, no cases of febrile neutropenia were reported. 11 (13\%) patients in the palbociclib + letrozole group and $2(2 \%)$ in the letrozole group discontinued the study because of adverse events. These results clearly show that inhibition of CDK4/6 is a promising 
way to improve the efficacy of endocrine treatment in ER-positive breast cancer patients with comparably few side effects.

Building on this substantial improvement of PFS with the addition of palbociclib to letrozole, the US Food and Drug Administration (FDA) granted accelerated approval to palbociclib (IBRANCE, Pfizer, Inc.) based on the designation as a 'breakthrough therapy' for use in combination with letrozole for the treatment of postmenopausal women with ER-positive, HER2-negative advanced breast cancer as initial endocrine-based therapy for their metastatic disease. The effectivity of palbociclib in hormone receptor-positive and HER2-negative advanced breast cancer was very recently confirmed in a phase 3 study (Paloma-3) with fulvestrant as backbone (results of PALOMA-3 are discussed in more detail by J. Ettl in this issue of BREAST CARE) [14].

\section{Beyond - Clinical Trials with Palbociclib in Progress}

A confirmatory phase 3 trial using palbociclib + letrozole in hormone receptor-positive and HER2-negative advanced-breast cancer patients, PALOMA-2, is fully recruited and the results are eagerly awaited. In addition, numerous trials of palbociclib in breast cancer are currently underway. ClinicalTrials.gov (https:// clinicaltrials.gov) was searched for a comprehensive listing of ongoing and completed studies. Search terms used were 'palbociclib' and 'breast cancer'. As of March 27, 2016, 39 studies were found using these search terms. Of these, 33 studies were open for recruitment. 2 of these studies had to be excluded (acute myeloid leukemia and squamous-cell lung cancer, respectively). Most of the trials are conducted in ER-positive advanced breast cancer and use an endocrine therapy (either aromatase inhibitor or fulvestrant or tamoxifen) as combination partner for palbociclib.

Beyond the well-known combination of palbociclib and endocrine therapies and bearing in mind the preclinical synergy of palbociclib and trastuzumab [8], several trials are currently conducted in HER2-positive breast cancer. The multicenter trial 'Neo-Adjuvant Treatment with Palbociclib: Effect on Ki67 and Apoptosis before, during and after Treatment' is currently recruiting ER- and HER2-positive patients (NCT02530424). Patients are treated with a neoadjuvant quadruplet (trastuzumab + pertuzumab + palbociclib + fulvestrant). The primary objectives of this phase 2 trial are changes of Ki-67 and apoptosis as well as the tolerability profile of the combination. A secondary objective is the rate of pathological complete response (pCR). The study of palbociclib and trastuzumab with or without letrozole in HER2-positive metastatic breast cancer (PATRICIA) (NCT02448420) is currently enrolling postmenopausal HER2-positive patients with advanced breast cancer. Patients who have already received chemotherapy and treatment with trastuzumab for their metastatic disease are treated with a combination of palbociclib + trastuzumab with or without letrozole. Depending on the hormone receptor status and treatment assignation, 3 patient cohorts are defined. Cohort A will include patients with ER-negative, HER2-positive breast cancer who will receive trastuzumab + palbociclib. Cohort B1 will include patients with ER-positive, HER2-positive breast cancer who will receive trastuzumab + palbociclib. Cohort B2 will include patients with ER-positive, HER2-positive breast cancer who will receive trastuzumab + palbociclib + letrozole. To avoid imbalance, assignation of ER-positive and HER2-positive patients to cohorts B1 and B2 will be randomized. Recruitment is planned for a maximum of 138 patients. The primary objective of this randomized phase 2 trial is PFS. Furthermore, a phase 1B inter-patient dose escalation study of palbociclib in combination with trastuzumab emtansine (T-DM1) in patients with recurrent or metastatic HER2-positive breast cancer after prior trastuzumab or other HER2-directed therapies is currently enrolling patients (NCT01976169). All patients will receive T-DM1 by intravenous infusion at $3.6 \mathrm{mg} / \mathrm{kg}$ for $90 \mathrm{~min}$ on day 1 of each 21-day cycle. A standard $3+3$ trial design will be used for palbociclib dose escalation cohorts. The primary outcome measures of this phase $1 \mathrm{~b}$ trial are MTD and DLT.

Preclinical data show that the combination of CDK4/6 inhibitors and phosphatidylinositol 3-kinase (PI3K) inhibitors overcomes intrinsic and adaptive resistance, leading to tumor regressions in phosphatidylinositol-4,5-bisphosphate 3-kinase catalytic subunit alpha (PIK3CA)-mutant breast cancer xenografts [15]. Based on these encouraging findings, a phase $1 \mathrm{~B}$ trial led by the Royal Marsden NHS Foundation Trust is combining the CDK4/6 inhibitor palbociclib with the PI3K inhibitors taselisib or pictilisib in ER-positive, HER2negative, PIK3CA-mutant breast cancer patients. (NCT02389842). During the dose escalation phase, there are 2 treatment arms with patients receiving either taselisib or pictilisib in combination with palbociclib. At the MTD dose expansion, fulvestrant will be administered in addition to palbociclib and taselisib or pictilisib. The primary outcome measure is the recommended dose for phase 2 .

However, at present, the combination of palbociclib with an endocrine therapy in hormone receptor-positive breast cancer is most thoroughly investigated and it is well accepted that efficacious new treatment options have the largest impact when used in early breast cancer. In this very setting, there are 2 large phase 3 trials actively recruiting participants.

First, the randomized post-neoadjuvant phase 3 trial PENELOPE-B led by the German Breast Group (GBG) is recruiting patients with increased risk of recurrence after neoadjuvant chemotherapy (NCT01864746). A post-neoadjuvant design is able to enrich the study population for patients with increased risk of recurrence. The PENELOPE-B trial will answer the question of whether palbociclib provides superior invasive disease-free survival (iDFS) compared to placebo in pre- and postmenopausal women with hormone receptor-positive, HER2-negative early breast cancer at high risk of relapse after showing less than $\mathrm{pCR}$ to neoadjuvant taxane-containing chemotherapy. The primary outcome measure is iDFS for palbociclib versus placebo in patients with high clinical/ pathological stage-ER status/tumor grade (CPS-EG) score after neoadjuvant chemotherapy receiving standard adjuvant endocrine therapy. This CPS-EG score was already validated to characterize hormone receptor-positive/HER2-negative patients with increased risk of recurrence after neoadjuvant chemotherapy [16]. The estimated enrollment of the PENELOPE-B trial is 1,100 patients. 
Second, the multicenter adjuvant phase 3 trial PALLAS is evaluating the addition of 2 years of palbociclib to standard adjuvant endocrine therapy for patients with hormone receptor-positive, HER2-negative early breast cancer (NCT02513394). Pre- and postmenopausal women or men with stage 2 or stage 3 hormone receptor-positive and HER2-negative early invasive breast cancer are eligible after breast surgery for the current malignancy. The primary outcome measure is iDFS. The expected enrollment of PALLAS is 4,600 patients.

In conclusion, inhibition of CDK4/6 using palbociclib in combination with endocrine therapies is an efficacious treatment op- tion in hormone receptor-positive/HER2-negative advanced breast cancer. Ongoing clinical trials will show whether there is also a role for this CDK4/6 inhibitor in HER2-positive breast cancer and whether palbociclib is ready for prime time in the adjuvant setting.

\section{Disclosure Statement}

M.S. reports personal fees from Astra Zeneca, Celgene, Eisai, Janssen, Novartis, Pfizer, Pierre-Fabre, Roche, Sividon, TEVA. In addition, M.S. has pending patents regarding the prediction of chemotherapeutic response in breast cancer and molecular markers for breast cancer prognosis.

\section{References}

1 Hanahan D, Weinberg RA: Hallmarks of cancer: the next generation. Cell 2011;144:646-674.

2 Malumbres M, Barbacid M: To cycle or not to cycle: a critical decision in cancer. Nat Rev Cancer 2001;1: 222-231.

3 Shapiro GI: Cyclin-dependent kinase pathways as targets for cancer treatment. J Clin Oncol 2006;24:17701783.

4 Bazarov AV, Lee WJ, Bazarov I, Bosire M, Hines WC, Stankovich B, Chicas A, Lowe SW, Yaswen P: The specific role of pRb in p16 (INK4A)-mediated arrest of normal and malignant human breast cells. Cell Cycle 2012;11:1008-1013.

5 Baughn LB, Di Liberto M, Wu K, Toogood PL, Louie T, Gottschalk R, Niesvizky R, Cho H, Ely S, Moore MAS, Chen-Kiang S: A novel orally active small molecule potently induces G1 arrest in primary myeloma cells and prevents tumor growth by specific inhibition of cyclin-dependent kinase 4/6. Cancer Res 2006;66: 7661-7667.

6 Fry DW, Harvey PJ, Keller PR, Elliott WL, Meade M, Trachet E, Albassam M, Zheng X, Leopold WR, Pryer NK, Toogood PL: Specific inhibition of cyclin-dependent kinase 4/6 by PD 0332991 and associated antitumor activity in human tumor xenografts. Mol Cancer Ther 2004;3:1427-1438.

7 Menu E, Garcia J, Huang X, Di Liberto M, Toogood PL, Chen I, Vanderkerken K, Chen-Kiang S: A novel therapeutic combination using PD 0332991 and bortezomib: study in the 5T33MM myeloma model. Cancer Res 2008;68:5519-5523.
Finn RS, Dering J, Conklin D, Kalous O, Cohen DJ, Desai AJ, Ginther C, Atefi M, Chen I, Fowst C, Los G, Slamon DJ: PD 0332991, a selective cyclin D kinase 4/6 inhibitor, preferentially inhibits proliferation of luminal estrogen receptor-positive human breast cancer cell lines in vitro. Breast Cancer Res 2009;11:R77.

9 Roberts PJ, Bisi JE, Strum JC, Combest AJ, Darr DB, Usary JE, Zamboni WC, Wong K, Perou CM, Sharpless NE: Multiple roles of cyclin-dependent kinase 4/6 inhibitors in cancer therapy. J Natl Cancer Inst 2012; 104:476-487.

10 Schwartz GK, LoRusso PM, Dickson MA, Randolph SS, Shaik MN, Wilner KD, Courtney R, O’Dwyer PJ: Phase I study of PD 0332991, a cyclin-dependent kinase inhibitor, administered in 3-week cycles (schedule 2/1). Br J Cancer 2011;104:1862-1868

11 Flaherty KT, Lorusso PM, Demichele A, Abramson VG, Courtney R, Randolph SS, Shaik MN, Wilner KD, O'Dwyer PJ, Schwartz GK: Phase I, dose-escalation trial of the oral cyclin-dependent kinase 4/6 inhibitor PD 0332991, administered using a 21-day schedule in patients with advanced cancer. Clin Cancer Res 2012; 18:568-576.

12 Demichele A, Clark AS, Tan KS, Heitjan DF, Gramlich K, Gallagher M, Lal P, Feldman M, Zhang P, Colameco C, Lewis D, Langer M, Goodman N, Domchek S, Gogineni K, Rosen M, Fox K, O’Dwyer P: CDK 4/6 inhibitor palbociclib (PD0332991) in Rb+ advanced breast cancer: phase II activity, safety, and predictive biomarker assessment. Clin Cancer Res 2015;21:9951001.
3 Finn RS, Crown JP, Lang I, Boer K, Bondarenko IM, Kulyk SO, Ettl J, Patel R, Pinter T, Schmidt M, Shparyk Y, Thummala AR, Voytko NL, Fowst C, Huang X, Kim ST, Randolph S, Slamon DJ: The cyclin-dependent kinase $4 / 6$ inhibitor palbociclib in combination with letrozole versus letrozole alone as first-line treatment of oestrogen receptor-positive, HER2-negative, advanced breast cancer (PALOMA-1/TRIO-18): a randomised phase 2 study. Lancet Oncol 2015;16:25-35.

14 Turner NC, Ro J, André F, Loi S, Verma S, Iwata H, Harbeck N, Loibl S, Huang Bartlett C, Zhang K, Giorgetti C, Randolph S, Koehler M, Cristofanilli M: Palbociclib in hormone-receptor-positive advanced breast cancer. N Engl J Med 2015;373:209-219.

15 Vora SR, Juric D, Kim N, Mino-Kenudson M, Huynh T, Costa C, Lockerman EL, Pollack SF, Liu M, Li X, Lehar J, Wiesmann M, Wartmann M, Chen Y, Cao ZA, Pinzon-Ortiz M, Kim S, Schlegel R, Huang A, Engelman JA: CDK 4/6 inhibitors sensitize PIK3CA mutant breast cancer to PI3K inhibitors. Cancer Cell 2014;26:136-149.

16 Marmé F, Lederer B, Blohmer J, Costa SD, Denkert C, Eidtmann H, Gerber B, Hanusch C, Hilfrich J, Huober J, Jackisch C, Kümmel S, Loibl S, Paepke S, Untch M, Minckwitz G von, Schneeweiss A: Utility of the CPS+EG staging system in hormone receptor-positive, human epidermal growth factor receptor 2-negative breast cancer treated with neoadjuvant chemotherapy. Eur J Cancer 2016;53:65-74. 